\title{
Aflatoxin exposure in children living in Mirpur, Dhaka: data from MAL-ED companion study
}

\author{
Mustafa Mahfuz ${ }^{1} \cdot$ Mohammed Ashraful Alam ${ }^{1} \cdot$ Shah Mohammad Fahim ${ }^{1} \cdot$ Md Amran Gazi $^{1}$. \\ Mohammad Jyoti Raihan ${ }^{1}$ - Muttaquina Hossain ${ }^{1}$ - Patricia A. Egner ${ }^{2}$ - Pascal Obong Bessong ${ }^{3}$ - William A. Petri ${ }^{4}$. \\ John D. Groopman ${ }^{2} \cdot$ Tahmeed Ahmed $^{1}$
}

Received: 9 September 2017 / Revised: 11 February 2018 / Accepted: 12 July 2018 / Published online: 5 September 2018

(c) The Author(s) 2018. This article is published with open access

\begin{abstract}
Dietary exposure to aflatoxin is implicated in growth faltering of children. Despite the high burden of childhood stunting in urban Bangladesh, there are no data on long-term exposure to aflatoxin. This study aimed to explore aflatoxin exposure levels in a group of children followed longitudinally. The current study used data and biospecimens collected during 20102014 as part of the MAL-ED birth cohort study in an urban slum of Mirpur, Dhaka where children were followed from birth to 36 months. AFB1-lysine adduct concentrations were determined by isotope dilution mass spectrometry from plasma samples collected at 7, 15, 24, and 36 months of age. The limit of detection was $0.5 \mathrm{pg}$ of AFB1-lys $/ \mathrm{mg}$ albumin. In 744 plasma samples, the geometric mean of AFB1-lysine/mg albumin was $1.07 \mathrm{pg}$ (range 0.04-123.5 pg/mg albumin). The proportion of children with detectable aflatoxin exposure was 10.1, 20.9, 17.9, and $61.7 \%$ for $7,15,24$, and 36 months, respectively. Reduction in breastfeeding prevalence (80\% at 24 months vs. $38 \%$ in 36 months) corresponded with the highlevel detection of AFB1-lysine at the age of 36 months. AFB1-lysine concentrations were the highest at the end of monsoon. This study reveals that $62 \%$ of children in slum settlement were exposed to aflatoxin by the end of the third year of life. High aflatoxin exposure was detected at the end of rainy season and with the introduction of family food. These findings suggest interventions to ameliorate the problem of chronic aflatoxin exposure including childhood stunting.
\end{abstract}

Keywords Aflatoxin $\cdot$ MAL-ED $\cdot$ Bangladesh $\cdot$ AFB1-lys $\cdot$ children

\section{Introduction}

Linear growth faltering in children is a pervasive public health burden for low- and middle-income countries globally, and it is a consequence of a wide variety of factors such as undernutrition, poor hygiene, low socioeconomic status, local political instability, and repeated episodes of infectious diseases [1]. In addition to the widely recognized

Mustafa Mahfuz

mustafa@icddrb.org

1 International Centre for Diarrhoeal Disease Research, Bangladesh (icddr,b), 68, Shaheed Tajuddin Ahmed Sarani, Mohakhali, Dhaka 1212, Bangladesh

2 Johns Hopkins Bloomberg School of Public Health, 615N. Wolfe Street, Baltimore, MD 21205, USA

3 University of Venda, Thohoyandou, South Africa

4 University of Virginia, Charlottesville, VA, USA risk factors, dietary and environmental toxins may have a potential role in childhood stunting [2, 3]. Reports from Sub-Saharan Africa have implicated aflatoxin exposure with stunting among children [2, 4-6]. However, childhood stunting is highly prevalent in South and East Asia, as well as Sub-Saharan Africa where food-borne aflatoxin exposure is high [2]. Bangladesh has one of the highest prevalence rates of childhood stunting in the world. About $36 \%$ of children under the age of 5 years in Bangladesh are stunted or short for their age, while $15 \%$ are reported to be severely stunted [7].

Aflatoxins are the secondary fungal metabolites of Aspergillus flavus, Aspergillus parasiticus, and occasionally other Aspergillus species. Aflatoxin contamination has been well-documented in African staple foods such as sorghum, groundnuts, and maize, but has also been demonstrated in a variety of other food commodities including rice, wheat, pulses, sorghum, dried fruits, and spices in tropical and subtropical regions [8]. Hot and humid environmental conditions increase the likelihood of the growth of 
Aspergillus spp. and subsequent toxin production [2]. The extreme weather events in Bangladesh are believed to be conducive for the growth of Aspergillus spp. [9]. Studies have documented aflatoxin contamination of different food commodities of Bangladesh [10, 11].

Despite the fact that there is limited data on the level of aflatoxin exposure in Bangladeshi people, a recent study conducted in the north of Bangladesh observed a high level of detectable aflatoxin in children aged 2 years or less [12]. Childhood stunting is extremely high in a slum setting and a recent study observed that $50 \%$ of under-5-year-old children in slums are stunted [13]. Given the high burden of childhood stunting, there are no data on long-term exposure to aflatoxin as measured by aflatoxin B1-lysine (AFB1-lys) adduct in plasma samples from children in urban Bangladesh.

Based on metabolic transformation, different exposure biomarkers for aflatoxin B1 (AFB1) have been developed from blood, urine, and tissue samples [14]. The biomarkers commonly used are metabolites, DNA-adducts, or protein adducts $[14,15]$. After ingestion, aflatoxin B1 is oxidized to highly reactive aflatoxin exo-epoxide by cytochrome P450 system in the liver. The epoxides then react with DNA to form guanine adducts or with serum albumin to generate lysine adducts [16]. DNA-adduct is a useful biomarker of AFB1 exposure but this method requires the availability of tissues to examine, which is a limitation [17]. The extent of the exposure of aflatoxin B1 can be measured, considering the dose-dependent manner of metabolites present in urine and blood [16]. Due to the short half-life, urine analysis can only be used in studies which detect acute exposure to AFB1 [17]. While AFB1lysine adduct has a half-life of 20 days, it reflects chronic exposure [16]. Moreover, AFB1-lysine adduct occurs in a dose-dependent manner and accumulates to a steady level that correlates with AFB1 DNA-adducts in internal organs $[17,18]$. Therefore, AFB1-lysine is the perfect less invasive biomarker of chronic aflatoxin exposure and has been used by almost all the studies conducted in the last decade.

This study was designed to explore the levels of aflatoxin exposure in a group of children followed longitudinally. This aflatoxin study took the advantage of a MAL-ED birth cohort study conducted in Bangladesh and used the framework of resources, data, and samples from MAL-ED study as it could control most of the variables associated with child growth and development [19]. "Etiology, Risk Factors and Interactions of Enteric Infections and Malnutrition and the Consequences for Child Health and Development" (MALED) is a multisite project aiming to gain a better understanding of the risk factors for malnutrition, enteric diseases, and associated health consequences, including developmental impairment, in children of developing countries.
MAL-ED is using a common research framework to test and develop hypotheses regarding enteric infections and, nutritional status and associations with childhood growth and development in eight developing countries including Bangladesh [20].

\section{Materials and methods}

\section{Study design and participants}

This MAL-ED aflatoxin study used data and biological samples from the MAL-ED birth cohort study in Bangladesh. In the MAL-ED birth cohort, the children were enrolled within 17 days of birth and followed longitudinally up to 36 months of age. Blood samples were collected at 7 , 15,24 , and 36 months of age [20]. The study site is located in the Bauniabadh area of Mirpur, Dhaka. This area is densely populated, inhabited by poor and lower-middleclass families, and has suboptimal sanitary conditions with a high burden of childhood under nutrition and infectious diseases. The study area is located in a hot and humid tropical climate zone with three different seasons: hot premonsoon season (March to May), rainy season or monsoon (June to October), and winter season from November to February [19].

A total of 212 children from the MAL-ED cohort were available and 196 of them were followed through 36 months of age. Only children with available plasma samples who provided consent to use their samples for aflatoxin assays were enrolled in this study. The research protocol was approved by the Institutional Review Board of icddr,b and informed written consent was obtained from one of the parents of each participant. A complete description of MAL-ED study methodology including description of site, recruitment, inclusion and exclusion criteria, sampling method, and IRB approvals has been published elsewhere [19-21].

\section{Aflatoxin plasma biomarker assay}

Aflatoxin B1-lysine adduct is a sensitive biomarker to determine long-term exposure to aflatoxin. All plasma samples were analyzed by an isotope dilution mass spectrometry method described in Groopman et al. [12]. In short, $200 \mu$ of sample was mixed with an internal standard $(10 \mu \mathrm{l} \times 0.1 \mathrm{ng}$ of AFB1-D4-lys $/ \mathrm{ml})$ and pronase solution (Millipore Corp. Catalog \#537088-100 $\mu \mathrm{M}$ ) and incubated for $18 \mathrm{~h}$ at $37^{\circ} \mathrm{C}$. Samples were passed through a solidphase extraction column (Waters Oasis MAX Cartridge 1 cc/30 mg, Catalog \#186000366) and analyzed by UPLC with mass spectrometric detection system. The internal standard parent molecular ion $((\mathrm{M}+\mathrm{H})+, \mathrm{m} / \mathrm{z} 461.3)$ 
fragments to yield a daughter ion at $\mathrm{m} / \mathrm{z}$ 398.2. The AFB1lys molecular ion ( $\mathrm{m} / \mathrm{z}$ 457.2) fragments to yield a daughter ion at $\mathrm{m} / \mathrm{z}$ 394.1. Three quality control samples from rats dosed with AFB1 were run daily. The limit of detection was $0.5 \mathrm{pg}$ of AFB1-lys/mg albumin.

\section{Surveillance and feeding data}

In the MAL-ED cohort study, illness and infant feeding data were collected through twice weekly home visits. Detailed information on dietary and morbidity surveillance was published by Richard S et al. [21]. Infant feeding practice and information on the consumption of breast milk was collected during biweekly visits. A complete description of the methodology on infant and child feeding practice was described by Caulfield et al. [20]. From month 9 to 36, a monthly 24-h recall method was used to collect quantitative data on child feeding and nutrient intakes [22].

\section{Socioeconomic status}

Based on preliminary research data, MAL-ED investigators had developed a simple composite index to measure the sum total of the socioeconomic status of families that can be compared across the countries and continents. This is known as the WAMI index. It includes water-sanitationhygiene, maternal education status, monthly income, and asset status. Complete description of the WAMI index has been well described elsewhere [23].

\section{Statistics}

In order to measure aflatoxin (AF), the blood sample was drawn when the sampled children were $7,15,24$, and 36 months old. Descriptive statistics was used to present the characteristics of the sample. Bivariate analyses were conducted to observe the effect of different food groups and other confounders on AF concentration. Multivariate analysis in the form of generalized estimating equation (GEE) was used to understand the contribution of different food items to AF concentration after adjusting for potential confounders. As the blood sample was drawn and $\mathrm{AF}$ was measured at four time points, the GEE approach ensured the controlling of the correlation between the measurements taken at different time points. Only those variables, whose statistically significant bivariate association with $\mathrm{AF}$ was confirmed, were regressed in the multivariate model. The multivariate model only had those samples whose AF was detectable during all time points. Statistical significance was declared when p-value was less than 0.05 and all analyses were conducted in STATA 13.0 (STATACorp.).

\section{Results}

In total, 228 children were enrolled in the study and 196 were followed up to 36 months of age. Blood samples were collected at 7, 15, 24, and 36 months of age. AFB1-lysine adduct (AFB1-lys) analysis was done from a total number of 744 plasma samples collected during 2010-2014 in four different time points ( 7 months, $n=208 ; 15$ months, $n=$ 196; 24 months, $n=173$; 36 months, $n=167)$. The percentages of aflatoxin detection samples at those time points were $10,20,17$, and $62 \%$, respectively. Figure 1 shows the study profile.

The baseline descriptive characteristics of the study are detailed in Table 1 . Nearly half of the study population was male and one in every five children had low birth weight. Around $16 \%$ of the children were stunted, $17 \%$ were wasted, and $21 \%$ were underweight at birth, indicating that the prevalence of undernutrition among the study samples was less than the national average. Table 2 and Fig. 2 show that the proportion of aflatoxin detection was similar in 15- and 24-months-old children. However, the lowest proportion of detection was for 7 months and the highest was for the 36months-old children. In addition to data on the prevalence of AF in different age groups (Fig. 2), we also obtained the prevalence of breastfeeding in the same time period. The proportion of breastfeeding decreased with increasing age. At 7 months, the prevalence of breastfeeding was $97 \%$, which decreased to $36 \%$ at 36 months.

Figure 3 shows the relationship between age, breastfeeding status, and AF detection status. The trend suggests an inverse relationship between breastfeeding status and AF detection. With increased age, the prevalence of breastfeeding decreases while the prevalence of AF detected increases. Moreover, between breastfed and non-breastfed infants across all four time points, aflatoxin detection was higher among non-breastfed infants (Table 3). Assessment of the seasonal influence on aflatoxin exposure is represented in Fig. 4. The prevalence of the highest AF detection was during August-October when any child turned 36 months old and the lowest was during May-July when children were 7 months old. In terms of seasonality of AF detection, the chi-square test dictates that there is no statistically significant difference between seasons for 7-, 15-, and 24-months-old children. However, there is a statistically significant difference $(p<0.001)$ in AF detection between seasons for the 36-months-old groups, with more detection in the months of August to October.

Bivariate analysis showed that most of the food items had statistically significant association with AF concentration except for yellow vegetables and organ meats. Gender, WAMI index, and maternal education status were also not statistically significant $(p>0.05)$ in the bivariate analyses. 
Fig. 1 Study profile

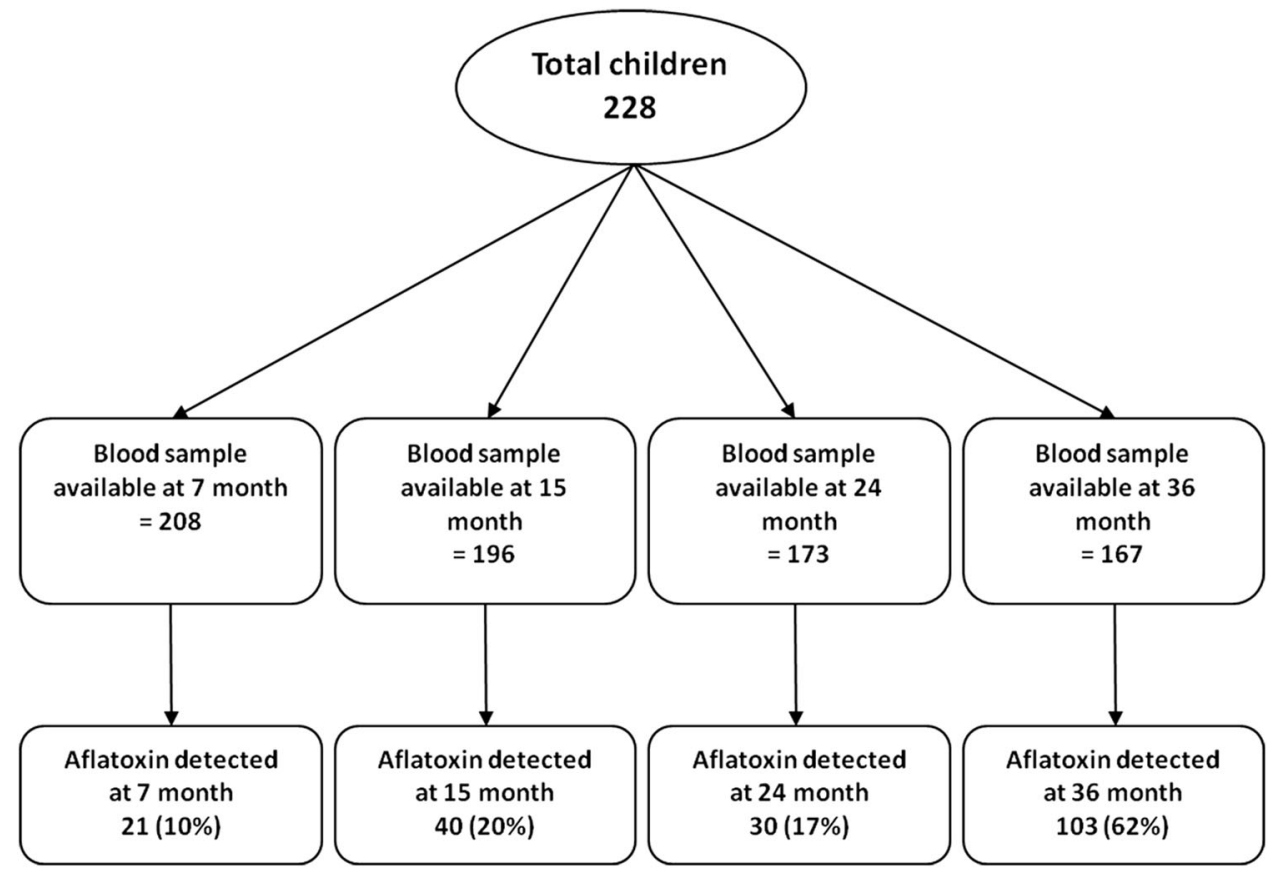

Table 1 Baseline characteristics of children in the aflatoxin (AF) study in Mirpur, Dhaka, Bangladesh $(n=229)$

\begin{tabular}{ll}
\hline Indicator & Value \\
\hline Male sex, $n(\%)$ & $108(47.58)$ \\
Low birth weight, $n(\%)$ & $50(21.83)$ \\
Cesarean delivery, $n(\%)$ & $47(20.70)$ \\
Breastfeeding started within $24 \mathrm{~h}, n(\%)$ & $218(95.20)$ \\
Weight for age $z$ score $<-2$ at birth, $n(\%)$ & $48(20.96)$ \\
Length for age $z$ score $<-2$ at birth, $n(\%)$ & $37(16.16)$ \\
Weight for length $z$ score $<-2$ at birth, $n(\%)$ & $37(17.05)$ \\
Mother's education $<5$ years, $n(\%)$ & $103(44.98)$ \\
Monthly family income in USD, median (IQR) & $101.26(75.95$, \\
& $126.58)$ \\
Number of food-insecure households, $n(\%)$ & $57(24.89)$ \\
Age at which the first solid food was given in & $173(154,187)$ \\
days, median (IQR) & \\
Duration of EBF in days, median (IQR) & $105(58,154)$ \\
WAMI index, mean \pm SD & $0.56 \pm 0.12$ \\
\hline
\end{tabular}

WAMI index: Composite score for water-sanitation-hygiene, maternal education status, monthly income, and asset status. Complete description of the WAMI index has been given by Psaki et al. [18]

However, in the multivariate model, only consumption of "sweets" was found to be significantly associated with AF [OR: 2.17 (95\% CI: 1.27, 3.70), $p<0.05$ ]. Both AugustOctober [OR: 3.09 (95\% CI: 1.83, 5.21) $p<0.05$ ] and November-January [OR: 2.03 (95\% CI: 1.16, 3.55), $p<$ $0.05]$ were significantly associated with $\mathrm{AF}$ concentration. Breastfeeding status [OR: 0.35 (95\% CI: 0.24, 0.52)] also had a significant protective effect against AF (Table 4).

\section{Discussion}

Chronic exposure to AF may be implicated in growth impairment which was evident in studies conducted in subSaharan Africa [4, 5, 24, 25]. But data on community-based estimation of AF exposure and its implication on childhood growth are very limited in South Asia. A study conducted in semi-urban neighborhood of Nepal demonstrated chronic AF exposure among children [26]. Another study performed in rural areas of Nepal and Bangladesh illustrates ubiquitous exposure of AF in both the countries [12]. Such type of chronic exposure may result in malnutrition and growth faltering in children. Most of the works related to this was done in African countries and very little is known about the AF exposure in urban South Asia. Our study is the first to explore the chronic exposure of AF in an urban slum of Mirpur, Bangladesh. In the present study, the geometric mean of plasma AFB1-lysine concentration at 36 months of age $(1.34 \mathrm{pg} / \mathrm{mg})$ was lower than the geometric mean of $4.06 \mathrm{pg} / \mathrm{mg}$, reported for the same-age children in Nepal. However, both the studies provide evidence of chronic aflatoxin exposure among children [26].

Previously, AFB1-alb biomarker by the ELISA method was commonly used for the detection of aflatoxin. But the advent of AFB1-lys albumin adducts biomarker made the detection of AF in dietary constituents more convenient. AFB1-lys pronase digestion product is typically measured by the LC-MS/MS method which is approximately 2.6 times more specific than AFB1-albumin measurement by ELISA. So, the different analytical methods that exist have varying sensitivities and specificity, which should be considered when 
Table 2 Summary statistics of aflatoxin study

\begin{tabular}{lllll}
\hline & & \multicolumn{2}{l}{ Bangladesh (229/265) } & \\
\cline { 4 - 5 } & Month-7 & Month-15 & Month-24 & Month-36 \\
\hline Number of blood samples collected & 212 & 197 & 177 & 168 \\
Number of insufficient samples & 4 & 1 & 4 & 1 \\
Number of available serum samples & 208 & 196 & 173 & 167 \\
Below the level of aflatoxin detected & 187 & 156 & 143 & 64 \\
Aflatoxin detected & $21(10.10 \%)$ & $40(20.41 \%)$ & $30(17.34 \%)$ & $103(61.68 \%)$ \\
Summary of AF lysine (pg/mg) & & & & \\
Mean & 1.30 & 1.52 & 3.43 & 3.70 \\
SD & 1.50 & 1.47 & 11.83 & 12.99 \\
Geometric mean & 0.71 & 0.94 & 0.98 & 1.34 \\
Median & 0.83 & 1.14 & 0.95 & 1.17 \\
IQR & $(0.29-1.56)$ & $(0.51-2.01)$ & $(0.57-1.23)$ & $(0.72-2.87)$ \\
Range & $(0.09-5.79)$ & $(0.06-6.35)$ & $(0.15-65.60)$ & $(0.09-126.54)$ \\
\hline
\end{tabular}

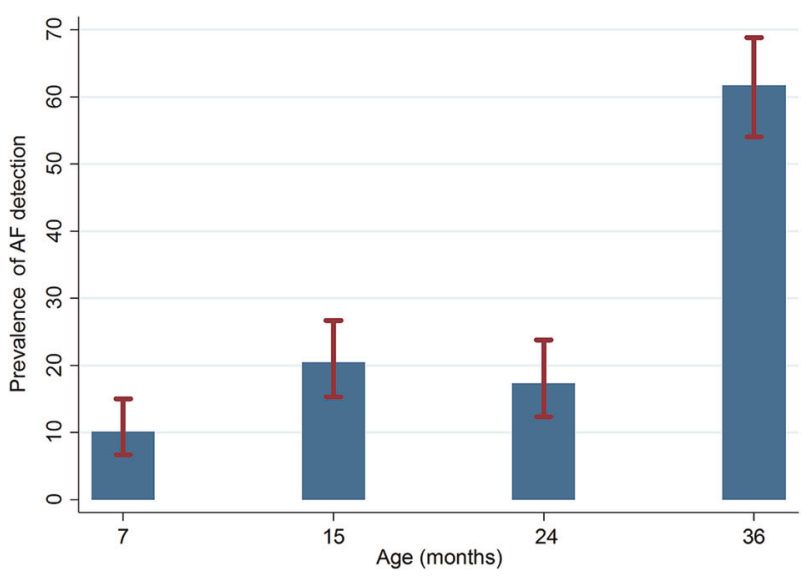

Fig. 2 Prevalence of AF detection

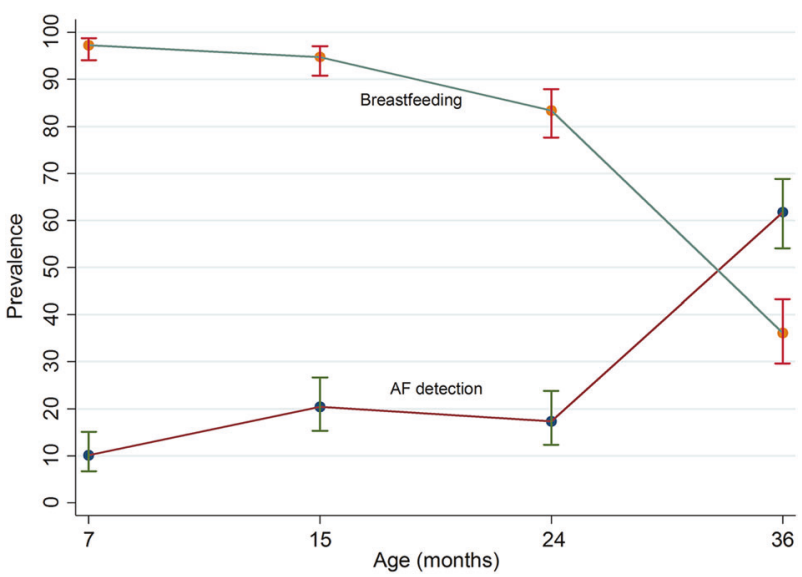

Fig. 3 Prevalence of breastfeeding and AF detection

making a comparison of AFB1-lys levels in different population-based studies worldwide. For instance, if we multiply the values for AFB1-lys in our study by 2.6, for comparative purposes with other aflatoxin-stunting studies that
Table 3 Breastfeeding status and aflatoxin detection

\begin{tabular}{lllll}
\hline Breastfeeding status & \multicolumn{4}{l}{ Aflatoxin detected ${ }^{\mathrm{a}}(\%, n)$} \\
\cline { 2 - 5 } & 7 months & 15 months & 24 months & 36 months \\
\hline Yes & $9.14(18)$ & $19.44(35)$ & $16.43(23)$ & $67.24(39)$ \\
No & $50(3)$ & $50(5)$ & $81.25(26)$ & $58.72(64)$ \\
$p$-value & $0.016^{\mathrm{b}}$ & $0.036^{\mathrm{b}}$ & 0.752 & 0.281 \\
\hline
\end{tabular}

${ }^{\mathrm{a}}$ Based on the limit of detection of $0.5 \mathrm{pg}$ of AFB1-lys/mg albumin bisher's exact test

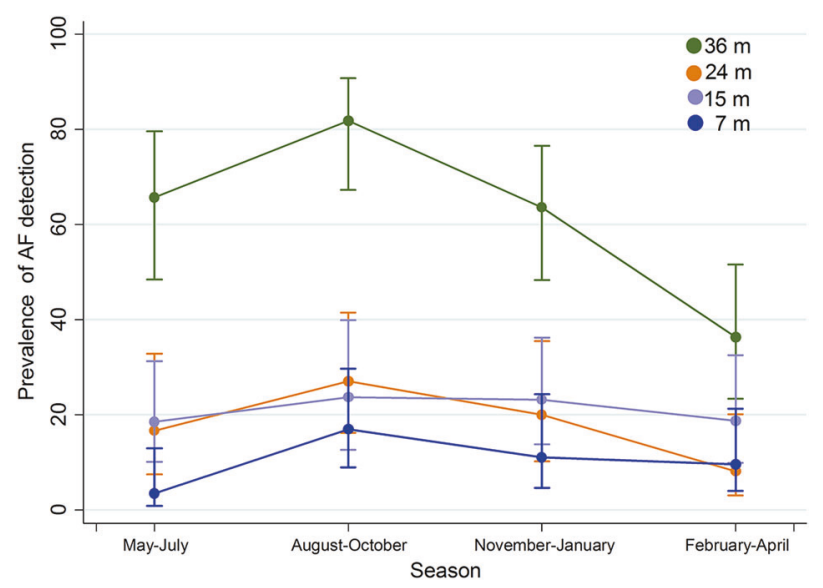

Fig. 4 Seasonal variation in AF detection while children were 7, 15, 24 , and 36 months old

all use ELISA methods, then the geometric mean is equivalent to 9.62 with a range of $0.234-329.004 \mathrm{pg} / \mathrm{mg}$ albumin. Although the mean is lower than that previously reported in African populations, the range is similar to those observed in one study conducted in 785 infants born in Ghana.

This study provides evidence that children in an urban slum of Mirpur are chronically exposed to aflatoxin from 7 to 36 months of age. AF exposure was the lowest at 
Table 4 Bivariate and multivariate relationships between aflatoxin exposure levels and variables related to socio-economic status, dietary intakes, maternal education, and seasonality

\begin{tabular}{|c|c|c|c|c|}
\hline & $\begin{array}{l}\text { Unadjusted odds ratio } \\
(95 \% \mathrm{CI})\end{array}$ & $p$-value & $\begin{array}{l}\text { Adjusted odds } \\
\text { ratio }^{\mathrm{e}}(95 \% \mathrm{CI})\end{array}$ & $p$-value \\
\hline Grain $^{\mathrm{a}}$ & $2.87(1.02,8.11)$ & 0.046 & $0.53(0.15,1.95)$ & 0.341 \\
\hline Root & $3.56(2.03,6.26)$ & 0.000 & $0.91(0.34,2.45)$ & 0.854 \\
\hline Yellow vegetable & $0.80(0.50,1.26)$ & 0.328 & & \\
\hline DGLV $^{\mathrm{b}}$ & $2.07(1.49,2.88)$ & 0.000 & $1.13(0.76,1.69)$ & 0.534 \\
\hline Legume & $3.41(1.98,5.87)$ & 0.000 & $1.41(0.58,3.44)$ & 0.448 \\
\hline Yellow fruits & $1.61(1.15,2.26)$ & 0.006 & $0.71(0.46,1.09)$ & 0.119 \\
\hline Other fruits and vegetables & $4.32(2.45,7.61)$ & 0.000 & $1.71(0.80,3.68)$ & 0.166 \\
\hline Meat & $2.01(1.44,2.80)$ & 0.000 & $1.21(0.80,1.83)$ & 0.358 \\
\hline Organ meat & $0.61(0.23,1.70)$ & 0.354 & & \\
\hline Egg & $2.83(1.97,4.06)$ & 0.000 & $1.58(0.97,2.58)$ & 0.065 \\
\hline Fish & $2.49(1.73,3.58)$ & 0.000 & $1.06(0.61,1.82)$ & 0.843 \\
\hline Dairy & $2.60(1.72,3.92)$ & 0.000 & $0.91(0.51,1.63)$ & 0.751 \\
\hline Sweet $^{\mathrm{c}}$ & $3.75(2.41,5.84)$ & 0.000 & $2.17(1.27,3.70)$ & 0.004 \\
\hline Breastfeeding & $0.26(0.18,0.38)$ & 0.000 & $0.34(0.23,0.50)$ & 0.000 \\
\hline Gender, female & $1.12(0.82,1.53)$ & 0.472 & & \\
\hline WAMI $^{\mathrm{d}}$ index & $0.70(0.18,2.81)$ & 0.619 & & \\
\hline \multicolumn{5}{|l|}{ Season (Ref: February-April) } \\
\hline May-July & $1.33(0.79,2.24)$ & 0.285 & $1.61(0.92,2.83)$ & 0.095 \\
\hline August-October & $2.69(1.66,4.37)$ & $<0.001$ & $3.02(1.79,5.11)$ & 0.000 \\
\hline November-January & $1.95(1.17,3.24)$ & 0.010 & $2.04(1.17,3.58)$ & 0.013 \\
\hline \multicolumn{5}{|c|}{ Mother's education (Ref: no education) } \\
\hline Primary incomplete & $1.03(0.62,1.73)$ & 0.902 & & \\
\hline Primary complete & $1.13(0.65,1.99)$ & 0.647 & & \\
\hline Secondary incomplete & $1.06(0.63,1.77)$ & 0.838 & & \\
\hline $\begin{array}{l}\text { Secondary complete or } \\
\text { higher }\end{array}$ & $0.96(0.42,2.16)$ & 0.916 & & \\
\hline
\end{tabular}

${ }^{a}$ Grains: rice, porridge, bread, noodles, or other foods/drinks made from grains

${ }^{b}$ DGLV: Dark green leafy vegetable

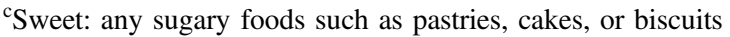

${ }^{\mathrm{d}}$ WAMI index: Composite score for water-sanitation-hygiene, maternal education status, monthly income, and asset status. Complete description of the WAMI index has been given by Psaki et al. [18]. Generalized estimating equation (GEE) was used to understand the contribution of different food items to AF concentration after adjusting for potential confounders

${ }^{\mathrm{e} O n l y}$ those variables, whose statistically significant bivariate association with AF was confirmed, were regressed in the multivariate model. The multivariate model only had those samples whose AF was detectable during all time points
7 months and it tends to increase with increasing age. The prevalence of AF exposure was the highest (62\%) at the end of the third year. At 7, 15, and 24 months of age, there were relatively lower percentages of detectable AF which is probably due to the major contribution of breast milk to the child's diet at this age. We assessed that the prevalence of breastfeeding reduced almost threefold (2.7 times) from 7 to 36 months of age. In contrast, AF exposure increased over the same time period with an increased introduction of family diet. Both the bivariate and multivariate analysis showed that reduction in breastfeeding is likely to be associated with an increase in aflatoxin exposure in Mirpur. This is in accordance with the findings of previous studies conducted in Sub-Saharan Africa, which reflected that weaning onto family food increases AF exposure [4, 5, 24, 25]. AF exposure is found to be significantly associated with weaning status in African studies because of the increases in consumption of maize and peanut-based food during the weaning period. It is noteworthy that even the children who were on partial breastfeeding had an increased level of AF-alb, possibly reflecting the increasing proportion of total food consumption coming from the weaning and family foods as the child becomes older [24, 25].

Dietary contamination of AF was detected in a variety of foodstuffs in Bangladesh [10, 11]. In consistence with that, $62 \%$ of serum samples were tested positive for AFB1-lys in 
our selected cohort. Most of the regularly consumed food items in Bangladesh including grain, legumes, meat, egg, and fish were found to be significantly associated with AF concentration except for yellow vegetables and organ meats. This finding correlates the most likely source of aflatoxin exposure during the weaning period. In the multivariate model, only "sweets" was found to be significantly associated with AF. As per our dietary data collection questionnaire, "sweet" food means any sugary food or drink that is sweet such as pastries, cakes, or biscuits. However, the major staple crop in this region is rice, which is relatively less susceptible to aflatoxin contamination. It was found that grain (that includes rice and wheat) has a significant association with $\mathrm{AF}$ contamination in our study at the bivariate level. But in Nepal, no association was demonstrated with the exposure of $\mathrm{AF}$ in relation to weaning status. It was reported from the MAL-ED cohort that Nepalese children had the highest consumption of formula feeding and solids/semisolid foods in the first month of their life. This early initiation of artificial feeding can be an explanation of no association between AF exposure and weaning status. Another potential reason can be the type of foods that is consumed in Nepal during the weaning period. Rice is the primary grain in Nepal which contains a very low amount of AF [26].

Our study result depicted the seasonal variation of AF with a highest detection during August-October among the children of 36 months. November-January was also significantly associated with $\mathrm{AF}$ exposure. $\mathrm{AF}$ is a potent mycotoxin that can contaminate staple grains in hot and humid climate. Bangladesh belongs to a subtropical climate zone with warm temperature and high humidity during monsoon and post-monsoon seasons [9]. Heavy rainfall and wet environment during monsoon provides an optimal condition for fungal growth and AF contamination, which may reveal immediately after the rainy season. Additionally, post-harvest storage of food crops in hot and humid conditions promotes mycotoxin formation and AF contamination in tropical countries [24]. In Bangladesh, the rainy season lasts from June to September and postmonsoon season extends up to October and November [9]. The increase in AFB1-lys levels during this period indicates the seasonal availability of aflatoxin contamination in staple grains. A similar type of seasonal variations was also observed in Nepal and African countries [5]. However, seasonal variation was not found significant for the children at 7,15 , and 24 months old.

There have been some limitations of the described study. First, this work would be more conclusive with additional dietary intake information and quantitative determination of the aflatoxin in those food items for each individual child. In fact, to fully distinguish the effects of the toxin from other confounding factors in the diet would require a randomized intervention study where the impact of lowering aflatoxin exposure on child immunity, growth, and disease susceptibility can be assessed. This would also permit a better understanding of the relative contribution of aflatoxin to growth impairment in relation to other important determinants in these communities.

In conclusion, the results of this study confirm that $62 \%$ of children are chronically exposed to aflatoxin in the third year of life. This is the first to report on AF in children from urban Bangladesh assessed by a robust mass spectrometrybased albumin adduct biomarker. High aflatoxin exposure was detected at the end of the rainy season and during the post-monsoon period. Reduction of breastfeeding and starting of family food was positively correlated with aflatoxin exposure. These data strongly suggest further exploration of the sources and determination of the threshold value of aflatoxin contamination in the staple grains in Bangladesh. However, the findings of this study point to critical windows for interventions to ameliorate the problem of chronic aflatoxin exposure and evaluate its impact on childhood growth. Further analysis will look into the association of aflatoxin exposure in relation to growth of young children in the same population.

\section{Availability of data}

Due to restriction in icddr,b's data access policy in regard to participants identifying information, data are available upon request from the Research \& Clinical Administration and Strategy (RCAS) of icddr,b (http://www.icddrb.org/ component/content/article/10003-data-policies/1893-datapolicies) for researchers who meet the criteria for access to confidential data.

Acknowledgements This research protocol is funded by the Bill \& Melinda Gates Foundation under its Global Health Program. BMGF Project investment id is OPP1136751. The sponsor was not involved in study design, data collection, analysis, interpretation of data, and writing the report. icddr,b acknowledges with gratitude the commitment of the Johns Hopkins University, University of Virginia, and University of Venda for their research efforts. The authors thank the staff and participants of the MAL-ED project and the MAL-ED Network investigators. icddr,b acknowledges the support of the MAL-ED consortium, particularly the Foundation of National Institute of Health (FNIH) and Fogarty International Centre (FIC). icddr,b also gratefully acknowledges the following donors who provide unrestricted support: Government of the People's Republic of Bangladesh; Global Affairs Canada (GAC); Swedish International Development Cooperation Agency (Sida); and the Department for International Development (UKAid).

Author contributions $\mathrm{MM}$ and TA originated the idea for the study and led the protocol design. MM, MAA, MH, POB, WP, JDG, and TA participated in the design of the study. MM, MAA, SMF, MAG, MJR, $\mathrm{MH}$, and TA conducted the study and supervised the sample and data collection. PAE and JG performed and supervised the sample analysis. 
MM, MAA, SMF, MAG, MJR, MH, and TA were involved in data analysis. MM, MJR, PAE, POB, WAP, JDG, and TA interpreted the results. MM, TA, MA, SMF, MAG, MJR, MH, PAE, POB, WP, JDG, and TA were involved in manuscript writing. All authors read and approved the final manuscript.

Funding The Bill and Melinda Gates Foundation.

\section{Compliance with ethical standards}

Conflict of interest The authors declare that they have no conflict of interest.

Open Access This article is licensed under a Creative Commons Attribution 4.0 International License, which permits use, sharing, adaptation, distribution and reproduction in any medium or format, as long as you give appropriate credit to the original author(s) and the source, provide a link to the Creative Commons license, and indicate if changes were made. The images or other third party material in this article are included in the article's Creative Commons license, unless indicated otherwise in a credit line to the material. If material is not included in the article's Creative Commons license and your intended use is not permitted by statutory regulation or exceeds the permitted use, you will need to obtain permission directly from the copyright holder. To view a copy of this license, visit http://creativecommons. org/licenses/by/4.0/.

\section{References}

1. Black RE, Allen LH, Bhutta ZA, Caulfield LE, de Onis M, Ezzati $\mathrm{M}$, et al. Maternal and child undernutrition: global and regional exposures and health consequences. Lancet. 2008;371:243-60.

2. Khlangwiset P, Shephard GS, Wu F. Aflatoxins and growth impairment: a review. Crit Rev Toxicol. 2011;41:740-55.

3. Wild CP. Aflatoxin exposure in developing countries: the critical interface of agriculture and health. Food Nutr Bull. 2007;28:S372-80.

4. Gong YY, Cardwell K, Hounsa A, Egal S, Turner PC, Hall AJ, et al. Dietary aflatoxin exposure and impaired growth in young children from Benin and Togo: cross sectional study. BMJ. 2002;325:20-1.

5. Gong Y, Hounsa A, Egal S, Turner PC, Sutcliffe AE, Hall AJ, et al. Postweaning exposure to aflatoxin results in impaired child growth: a longitudinal study in Benin, West Africa. Environ Health Perspect. 2004;112:1334-8.

6. Kensler TW, Roebuck BD, Wogan GN, Groopman JD. Aflatoxin: a 50-year odyssey of mechanistic and translational toxicology. Toxicol Sci. 2011;120:S28-48.

7. National Institute of Population Research and Training (NIPORT), Mitra and Associates, MEASURE DHS-ICF International. Bangladesh demographic and health survey 2011: preliminary Report. Dhaka, Bangladesh and Calverton, MD: National Institute of Population Research and Training, Mitra and Associates and ICF International; 2012.

8. Strosnider H, Azziz-Baumgartner E, Banziger M, Bhat RV, Breiman R, Brune MN, et al. Workgroup report: public health strategies for reducing aflatoxin exposure in developing countries. Environ Health Perspect. 2006;114:1898-903.

9. Shahid S. Probable impacts of climate change on public health in Bangladesh. Asia Pac J Public Health. 2010;22:310-9.

10. Dawlatana M, Coker RD, Nagler MJ, Wild CP, Hassan MS, Blunden $\mathrm{G}$. The occurrence of mycotoxins in key commodities in
Bangladesh: surveillance results from 1993 to 1995. J Nat Toxins. 2002;11:379-86.

11. Roy M, Harris J, Afreen S, Deak E, Gade L, Balajee SA, et al. Aflatoxin contamination in food commodities in Bangladesh. Food Addit Contam Part B Surveill. 2013;6:17-23.

12. Groopman JD, Egner PA, Schulze KJ, Wu LS, Merrill R, Mehra $\mathrm{S}$, et al. Aflatoxin exposure during the first 1000 days of life in rural South Asia assessed by aflatoxin $\mathrm{B}_{1}$-lysine albumin biomarkers. Food Chem Toxicol. 2014;74:184-9.

13. Ahsan KZ, Arifeen SE, Al-Mamun MA, Khan SH, Chakraborty N. Effects of individual, household and community characteristics on child nutritional status in the slums of urban Bangladesh. Arch Public Health. 2017;75:9.

14. Ahmed T, Mahfuz M, Islam MM, Mondal D, Hossain MI, Ahmed AS, et al. The MAL-ED Cohort Study in Mirpur, Bangladesh. Clin Infect Dis. 2014;59:S280-6.

15. MAL-ED Network Investigators.. The MAL-ED Study: a multinational and multidisciplinary approach to understand the relationship between enteric pathogens, malnutrition, gut physiology, physical growth, cognitive development, and immune responses in infants and children up to 2 years of age in resource-poor environments. Clin Infect Dis. 2014;59:S193-206.

16. Richard SA, Barrett LJ, Guerrant RL, Checkley W, Miller MA. MAL-ED Network Investigators. Disease surveillance methods used in the 8-Site MAL-ED Cohort Study. Clin Infect Dis. 2014;59:S220-4.

17. Caulfield LE, Bose A, Chandyo RK, Nesamvuni C, de Moraes ML, Turab A, et al. Infant feeding practices, dietary adequacy, and micronutrient status measures in the MAL-ED study. Clin Infect Dis. 2014;59:S248-54.

18. Psaki SR, Seidman JC, Miller M, Gottlieb M, Bhutta ZA, Ahmed $\mathrm{T}$, et al. Measuring socioeconomic status in multicountry studies: results from the eight-country MAL-ED study. Popul Health Metr. 2014; $12: 8$.

19. Turner PC, Moore SE, Hall AJ, Prentice AM, Wild CP. Modification of immune function through exposure to dietary aflatoxin in Gambian children. Environ Health Perspect. 2003;111: 217-20.

20. Egal S, Hounsa A, Gong YY, Turner PC, Wild CP, Hall AJ, et al. Dietary exposure to aflatoxin from maize and groundnut in young children from Benin and Togo, West Africa. Int J Food Microbiol. 2005;104:215-24.

21. Mitchell NJ, Hsu HH, Chandyo RK,4, Shrestha B, Bodhidatta L, $\mathrm{Tu} \mathrm{YK}$, et al. Aflatoxin exposure during the first 36 months of life was not associated with impaired growth in Nepalese children: an extension of the MAL-ED study. PLoS ONE. 2017;12: $\mathrm{e} 0172124$.

22. Mitchell NJ, Riley RT, Egner PA, Groopman JD, Wu F. Chronic aflatoxin exposure in children living in Bhaktapur, Nepal: extension of theMAL-ED study. J Exp Sci Environ Epidemiol. 2017;27:106-11

23. Scholl PF, Turner PC, Sutcliffe AE, Sylla A, Diallo MS, Friesen $\mathrm{MD}$, et al. Quantitative comparison of aflatoxin B1 plasma albumin adducts in humans by isotope dilution mass spectrometry and ELISA. Cancer Epidemiol Biomark Prev. 2006;15:823-6.

24. Aidoo KE. Post-harvest storage and preservation of tropical crops. Int Biodeterior Biodegrad. 1993;32:161-73.

25. Turner PC, Mendy M, Whittle H, Fortuin M, Hall AJ, Wild CP. Hepatitis B infection and aflatoxin biomarker levels in Gambian children. Trop Med Int Health. 2000;5:837-41.

26. Turner PC, Sylla A, Gong YY, Diallo MS, Sutcliffe AE, Hall AJ, et al. Reduction in exposure to carcinogenic aflatoxins by postharvest intervention measures in West Africa: a community-based intervention study. Lancet. 2005;365:1950-6. 